\title{
Exhausted Plume Flow Field Prediction Near the Afterbody of Hypersonic Flight Vehicles in High Altitudes
}

\author{
Zheng-Tao Deng $\dagger$, Goang-Shin Liaw $\ddagger$ \\ Department of Civil Engineering \\ Alabama A\&M University \\ Huntsville, Alabama 35762 \\ Lynn Chen Chou* \\ NASA/Marshall Space Flight Center \\ Huntsville, AL 35812 \\ Kervyn D. Mach* \\ Wright-Patterson Air Force Base \\ Dayton, Ohio 45439
}

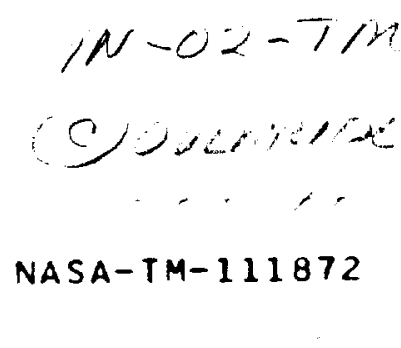

\section{ABSTRACT}

A two-dimensional computer code to solve the Burnett equations has been developed which computes the flow interaction between an exhausted plume and hypersonic external flow near the afterbody of a flight vehicle. This Burnett-2D code extends the capability of Navier-Stokes solver (RPLUS2D code) to include highorder Burnett source terms and slip-wall conditions for velocity and temperature. Higher-order Burnett viscous stress and heat flux terms are discretized using centraldifferencing and treated as source terms. Blocking logic is adopted in order to overcome the difficulty of grid generation. The computation of exhaust plume flow field is divided into two steps. In the first step, the thruster nozzle exit conditions are computed which generates inflow conditions in the base area near the afterbody. Results demonstrated that at high altitudes, the computations of nozzle exit conditions must include the effects of base flow since significant expansion exists in the base region. In the second step, Burnett equations were solved for exhaust plume flow field near the afterbody. The free stream conditions are set at an altitude equal to $80 \mathrm{~km}$ and the Mach number is equal to 5.0. The preliminary results show that the plume expansion, as altitudes increases, will eventually cause upstream flow separation.

\section{INTRODUCTION}

For a single-stage-to-orbit hypersonic flight vehicle cruising at high altitudes, an exhaust plume is created in the base area by a propulsive device. This plume interacts with the free stream near the afterbody and may create the undesirable plume-induced separation. This separation affects the dynamic characteristics of the flight

$\dagger$ Assistant Professor, Member AIAA

$\ddagger$ Professor, Member AIAA

* Senior Member AIAA

Copyright (c) American Institute of Aeronautics and Astronautics. Inc.. 1995. All rights reserved. vehicle. Due to the difficulty of collecting reliable windtunnel data, numerically simulated data has become imperative for this purpose.

As the flight altitude increases from sea level to about $70 \mathrm{~km}$, the plume/free-stream interaction flow field is in the continuum regime. The conventional NavierStokes equations are valid governing equations. As altitude increases from 70 to $100 \mathrm{~km}$, the ambient air becomes more rarefied and the flow is usually in the transitional regime. The transitional flow regime is defined when the Knudsen number $K n$ is from 0.01 to 1.0. The prevailing reason for the failure of applying the Navier-Stokes equations in the transitional flow regime is primarily due to the breakdown of the translational equilibrium ${ }^{[1,2]}$. It is common to apply the Direct Simulation Monte Carlo (DSMC) method for rarefied gasdynamic problems. Moss ${ }^{[3]}$ conducted a series of DSMC analysis and concluded that the Navier-Stokes equations cease to be valid above 92 kilometers. The drawback of the DSMC calculation for flow problems with small Knudsen number is that the computational time becomes prohibitively expensive.

To circumvent these discrepancies and to preserve the continuum modeling, the third-order Burnett equations are hypothesized as the governing equations in the transitional regime. Zhong ${ }^{[4,5]}$ conducted a series of hypersonic flow analysis for flow over a blunt body using the Augmented Burnett equations. Liaw, Deng and Chou ${ }^{[6,7,8]}$ also conducted a series of analysis for shock wave structure prediction and low-density nozzle flow calculations to solve the Burnett and Boltzmann equations. It was concluded that the Burnett solutions are more accurate than the Navier-Stokes solutions in the transitional regime.

The objective of the current research is to numerically investigate the exhausted plume flow field near the afterbody of a hypersonic flight vehicle in high altitudes by solving the Burnett equations.

\section{MATHEMATICAL FORMULATIONS}

The Burnett equations are derived to include the second-order term of the distribution function in the Chapman-Enskog expansion series, 


$$
f=f_{0}+K \operatorname{Lin} f_{1}+\operatorname{Lin}^{2} f_{2}+\cdots,
$$

so that the Knudsen number effect is emphatically embedded into the moment equations. As far as the mathematical manipulation is concerned, the Navier-Stokes equations are the subset of the Burnett equations because the Navier-Stokes equations are obtained with the first order Chapman-Enskog expansion. In consequence, the Burnett equations are a system of third-order nonlinear partial differential equations which are far more complicated than the Navier-Stokes equations.

Written in a general body-fitted coordinate system, $(\xi, \eta)$, the two-dimensional Burnett equations can be expressed as

$$
\frac{\partial \dot{Q}}{\partial \tau}+\frac{\partial}{\partial \xi}\left(\dot{E}-\dot{E}_{v}\right)+\frac{\partial}{\partial \eta}\left(\dot{F}-\hat{F}_{v}\right)=0
$$

where

$$
\begin{aligned}
& \hat{Q}=H \cdot\left(\begin{array}{c}
\rho \\
\rho u \\
\rho v \\
\rho e_{t}
\end{array}\right)_{4 \times 1} \\
& \dot{E}=H \cdot\left(\xi_{x}\left(\begin{array}{c}
\rho u \\
\rho u^{2}+p \\
\rho u v \\
u\left(\rho e_{t}+p\right)
\end{array}\right)+\xi_{y}\left(\begin{array}{c}
\rho v \\
\rho u v \\
\rho v^{2}+p \\
v\left(\rho e_{t}+p\right)
\end{array}\right)\right) \\
& \hat{F}=H \cdot\left(\eta_{x}\left(\begin{array}{c}
\rho u \\
\rho u^{2}+p \\
\rho u v \\
u\left(\rho e_{t}+p\right)
\end{array}\right)+\eta_{y}\left(\begin{array}{c}
\rho v \\
\rho u v \\
\rho v^{2}+p \\
v\left(\rho e_{t}+p\right)
\end{array}\right)\right) \\
& \hat{E}_{v}=H \xi_{x}\left(\begin{array}{c}
0 \\
\tau_{x x}+\tau_{x x}^{(2)} \\
\tau_{x y}+\tau_{x y}^{(2)} \\
u\left(\tau_{x x}+\tau_{x x}^{(2)}\right) \\
+v\left(\tau_{x y}+\tau_{x y}^{(2)}\right)-q_{x}-q_{x}^{(2)}
\end{array}\right)_{4 \times 1} \\
& +H \xi_{y}\left(\begin{array}{c}
0 \\
\tau_{y x}+\tau_{y x}^{(2)} \\
\tau_{y y}+\tau_{y y}^{(2)} \\
u\left(\tau_{y x}+\tau_{y x}^{(2)}\right) \\
+v\left(\tau_{y y}+\tau_{y y}^{(2)}\right)-q_{y}-q_{y}^{(2)}
\end{array}\right)_{4 \times 1} \\
& \dot{F}_{v}=H \eta_{x}\left(\begin{array}{c}
0 \\
\tau_{x x}+\tau_{x x}^{(2)} \\
\tau_{x y}+\tau_{x y}^{(2)} \\
u\left(\tau_{x x}+\tau_{x x}^{(2)}\right) \\
+v\left(\tau_{x y}+\tau_{x y}^{(2)}\right)-q_{x}-q_{x}^{(2)}
\end{array}\right)_{4 \times 1} \\
& +H \eta_{y}\left(\begin{array}{c}
0 \\
\tau_{y x}+\tau_{y x}^{(2)} \\
\tau_{y y}+\tau_{y y}^{(2)} \\
u\left(\tau_{y x}+\tau_{y x}^{(2)}\right) \\
+v\left(\tau_{y y}+\tau_{y y}^{(2)}\right)-q_{y}-q_{y}^{(2)}
\end{array}\right)_{4 \times 1}
\end{aligned}
$$

In equations (3),(4), (5), (6) and (7), $H$ is the cell volume, $\rho$ is density $u$ and $u$ are velocity components in Cartesian

coordinates $(x, y), p$ is pressure, $e_{t}$ is the specific total energy, $\tau_{x x}, \tau_{x y}, \tau_{y y}$ are the Navier-Stokes shear stress components, $\tau_{x x}^{(2)}, \tau_{x y}^{(2)}, \tau_{y y}^{(2)}$ are the Burnett stress components, $q_{x}, q_{y}$ are the first-order heat flux components. and $q_{x}^{(2)}, q_{y}^{(2)}$ are the Burnett heat flux components.

The Burnett stress components $\left(\tau_{x x}^{(2)}, \tau_{x y}^{(2)}, \tau_{y x}^{(2)}, \tau_{y y}^{(2)}\right)$, and heat flux components $\left(q_{x}^{(2)}, q_{y}^{(2)}\right)$, are derived from the previous work ${ }^{[8]}$. In two-dimensional Cartesian coordinates $(x, y)$, they can be expressed as

$$
\begin{aligned}
\tau_{x x}^{(2)}=\frac{\mu^{2}}{p} & \left(\alpha_{1} u_{x}^{2}+\alpha_{2} u_{x} v_{y}+\alpha_{3} v_{y}^{2}+\alpha_{4} u_{y} v_{x}\right. \\
& +\alpha_{5} u_{y}^{2}+\alpha_{6} v_{x}^{2}+\alpha_{7} R T_{x x}+\alpha_{8} R T_{y y} \\
& +\alpha_{9} \frac{R T}{\rho} \rho_{x x}+\alpha_{10} \frac{R T}{\rho} \rho_{y y}+\alpha_{11} \frac{R T}{\rho^{2}} \rho_{x}^{2} \\
& +\alpha_{12} \frac{R}{\rho} T_{x} \rho_{x}+\alpha_{13} \frac{R}{T} T_{x}^{2}+\alpha_{14} \frac{R T}{\rho^{2}} \rho_{y}^{2} \\
& \left.+\alpha_{15} \frac{R}{\rho} T_{y} \rho_{y}+\alpha_{16} \frac{R}{T} T_{y}^{2}\right)
\end{aligned}
$$$$
\tau_{y y}^{(2)}=\frac{\mu^{2}}{p}\left(\alpha_{1} v_{y}^{2}+\alpha_{2} v_{y} u_{x}+\alpha_{3} u_{x}^{2}+\alpha_{4} v_{x} u_{y}\right.
$$$$
+\alpha_{5} v_{x}^{2}+\alpha_{6} u_{y}^{2}+\alpha_{7} R T_{y y}+\alpha_{8} R T_{x x}
$$$$
+\alpha_{9} \frac{R T}{\rho} \rho_{y y}+\alpha_{10} \frac{R T}{\rho} \rho_{x x}+\alpha_{11} \frac{R T}{\rho^{2}} \rho_{y}^{2}
$$$$
+\alpha_{12} \frac{R}{\rho} T_{y} \rho_{y}+\alpha_{13} \frac{R}{T} T_{y}^{2}+\alpha_{14} \frac{R T}{\rho^{2}} \rho_{x}^{2}
$$$$
\left.+\alpha_{15} \frac{R}{\rho} T_{x} \rho_{x}+\alpha_{16} \frac{R}{T} T_{x}^{2}\right)
$$$$
\begin{aligned}
\tau_{x y}^{(2)}=\frac{\mu^{2}}{p} & \left(\beta_{1} u_{x} u_{y}+\beta_{2} u_{y} v_{y}+\beta_{2} u_{x} v_{x}+\beta_{1} v_{x} v_{y}\right. \\
& +\beta_{3} R T_{x y}+\beta_{4} \frac{R T}{\rho} \rho_{x y}+\beta_{5} \frac{R}{T} T_{x} T_{y} \\
& \left.+\beta_{6} \frac{R T}{\rho^{2}} \rho_{x} \rho_{y}+\beta_{7} \frac{R}{\rho} \rho_{x} T_{y}+\beta_{7} \frac{R}{\rho} \rho_{y} T_{x}\right)
\end{aligned}
$$$$
q_{x}^{(2)}=\frac{\mu^{2}}{\rho}\left(\frac{\gamma_{1}}{T} u_{x} T_{x}+\frac{\gamma_{2}}{T} T_{x} v_{y}+\gamma_{3} u_{x x}\right.
$$$$
+\gamma_{4} u_{y y}+\gamma_{5} v_{x y}+\frac{\gamma_{6}}{T} T_{y} v_{x}
$$$$
+\frac{\gamma_{7}}{T} T_{y} u_{y}+\frac{\gamma_{8}}{\rho} \rho_{x} u_{x}+\frac{\gamma_{9}}{\rho} \rho_{x} v_{y}
$$$$
\left.+\frac{\gamma_{10}}{\rho} \rho_{y} u_{y}+\frac{\gamma_{11}}{\rho} \rho_{y} v_{x}\right)
$$

$$
\begin{aligned}
q_{y}^{(2)}=\frac{\mu^{2}}{\rho} & \left(\frac{\gamma_{1}}{T} T_{y} v_{y}+\frac{\gamma_{2}}{T} T_{y} u_{x}+\gamma_{3} v_{y y}\right. \\
& +\gamma_{4} v_{x x}+\gamma_{5} u_{x y}+\frac{\gamma_{6}}{T} T_{x} u_{y} \\
& +\frac{\gamma_{7}}{T} T_{x} v_{x}+\frac{\gamma_{8}}{\rho} \rho_{y} v_{y}+\frac{\gamma_{9}}{\rho} \rho_{y} v_{x} \\
& \left.+\frac{\gamma_{10}}{\rho} \rho_{x} v_{x}+\frac{\gamma_{11}}{\rho} \rho_{x} u_{y}\right)
\end{aligned}
$$


where, the subindex $x$ and $y$ represent the partial derivatives with respect to coordinates $x$ and $y$, e.g.,

$$
\begin{gathered}
T_{x}=\frac{\partial T}{\partial x} \quad T_{y}=\frac{\partial T}{\partial y} \quad T_{x y}=\frac{\partial^{2} T}{\partial x \partial y} \\
T_{x x}=\frac{\partial^{2} T}{\partial x^{2}} \quad T_{y y}=\frac{\partial^{2} T}{\partial y^{2}}
\end{gathered}
$$

The coefficients $\alpha_{i}, \beta_{i}, \gamma_{i}$ are obtained based on the Chapman-Enskog expansion and are depend on the molecular force model ${ }^{[8]}$. In this research, the Maxwellian gas model is assumed.

\section{NUMERICAL PROCEDURES}

Numerical Scheme for Burnett Equations. The solution methodology for two-dimensional Burnett equations is extended from the RPLUS2D ${ }^{[9]}$ code. The second-order upwind (UP) scheme, namely, the StegerWarming vector splitting scheme, and the Lower-Upper (LU) approximate factorization methods are used to discretize the Burnett equations. The discretized Burnett equations can be written as

$$
\begin{gathered}
\left(I+\Delta \tau\left(\Delta_{\xi}^{-} \hat{A}_{i, j}^{+}+\Delta_{\xi}^{+} \hat{A}_{i, j}^{-}+\Delta_{\eta}^{-} \hat{B}_{i, j}^{+}+\Delta_{\eta}^{+} \hat{B}_{i, j}^{-}\right) \delta \hat{Q}\right. \\
=-\Delta \tau \operatorname{Res} s_{i, j}
\end{gathered}
$$

where, the right-hand-side residue $\operatorname{Res}_{i, j}$ is

$$
\operatorname{Res}_{i, j}=\frac{\partial}{\partial \xi}\left(\hat{E}-\hat{E}_{v}\right)+\frac{\partial}{\partial \eta}\left(\hat{F}-\hat{F}_{v}\right)
$$

In equations (13) and (14), $\Delta^{+}$and $\Delta^{-}$are forward and backward one-sided differencing operators, and

$$
\begin{aligned}
& \hat{A}=\frac{\partial \hat{E}}{\partial \hat{Q}}=\hat{A}^{+}+\hat{A}^{-}=S_{\xi}\left(\Lambda_{\xi}^{+}+\Lambda_{\xi}^{-}\right) S_{\xi}^{-1} \\
& \hat{B}=\frac{\partial \hat{F}}{\partial \hat{Q}}=\hat{B}^{+}+\hat{B}^{-}=S_{\eta}\left(\Lambda_{\eta}^{+}+\Lambda_{\eta}^{-}\right) S_{\eta}^{-1}
\end{aligned}
$$

in which $\Lambda_{\xi}^{+}, \Lambda_{\eta}^{+}, \ldots$ etc. are the diagonal eigen-value matrices, and $S_{\xi}$ etc. are the right eigen-matrices obtained from the Steger-Warming splitting. Substituting $\Delta^{+}$and $\Delta^{-}$into equation (14), it becomes

$$
(D+L+U) \delta \hat{Q}=-\Delta t \operatorname{Res}_{i, j}
$$

where

$$
\begin{gathered}
D=I+\Delta t\left(\hat{A}_{i, j}^{+}-\hat{A}_{i, j}^{-}+\hat{B}_{i, j}^{+}-\hat{B}_{i, j}^{-}\right) \\
L=-\Delta t\left(\hat{A}_{i-1, j}^{+}+\hat{B}_{i, j-1}^{+}\right) \\
U=\Delta t\left(\hat{A}_{i+1, j}^{-}+\hat{B}_{i, j+1}^{-}\right)
\end{gathered}
$$

After applying approximate factorization procedures, it becomes

$$
(D+L) D^{-1}(D+U) \delta \dot{Q}_{i, j}=-\Delta t \operatorname{Res}_{i, j}
$$

where $D$ is a diagonal matrix, $L$ and $U$ are upper and lower matrices, respectively. At each time step, the operation of LU-SW/UP scheme can be written as:

$$
\begin{aligned}
(D+L) \delta \hat{Q}_{i, j}^{*} & =-\Delta t \operatorname{Res}_{i, j} \\
(D+U) \delta \hat{Q}_{i, j} & =D \delta \hat{Q}_{i, j}^{*} \\
\hat{Q}_{i, j}^{n+1} & =\hat{Q}_{i, j}^{n}+\delta \hat{Q}_{i, j}
\end{aligned}
$$

For residual $\left(\operatorname{Res}_{i, j}\right)$ calculations, the Osher's upwind differencing scheme is used for inviscid terms, and central differencing is used for viscous and Burnett terms.

$$
\begin{aligned}
\operatorname{Res}_{i, j}= & H\left(\frac{\partial \dot{E}}{\partial \xi}+\frac{\partial \dot{F}}{\partial \eta}\right)_{\text {osher }} \\
& +H\left(\left(\hat{E}_{v}\right)_{i+1 / 2, j}-\left(\hat{E}_{v}\right)_{i-1 / 2, j}\right) \\
& +H\left(\left(\hat{F}_{v}\right)_{i, j+1 / 2}-\left(\hat{F}_{v}\right)_{i, j-1 / 2}\right)
\end{aligned}
$$

The Burnett stress and heat flux terms are treated as source terms in the solution procedure. At each time step, the source terms are calculated explicitly based on flow parameters of the previous time step. A timemarching technique is used to obtain the steady state solutions. A steady state solution is obtained when all residues (density, velocity and enthalpy) are less than $10^{-6}$.

Blocking Algorithm and Boundary Conditions. To reduce the difficulty of grid generation, the blocking logic was used to compute the flow near the afterbody. The computational domain is divided into two blocks of grid system as shown in Figure 1. The first block is constructed in the lower part of the afterbody. The second block is constructed in the plume region near the afterbody. For block-one, free stream, wall, interface and supersonic outflow boundary conditions must be specified for equilibrium air flow. For block-two, thruster nozzle exit conditions, wall, interface and outflow conditions need to be given. The thruster nozzle exit conditions were computed independently using the RPLUS2D code. The nozzle calculation is assumed a $\mathrm{H}_{2} / \mathrm{O}_{2}$ equilibrium flow. The base flow area is included in the computational domain and the flow field in the base area is computed simutaneously with the nozzle internal flow field. The computed nozzle exit conditions are then averaged and used as the inflow boundary for block-two. The interface conditions have to be coupled between block-one and block-two. A first-order upwind approximation scheme is applied at the interface. Since the conventional continúum no-slip wall conditions break down as the flow becomes rarefied, the slip-wall conditions are applied at the wall. Based on the first-order Maxwell/Smoluchowshi slip boundary conditions, the wall temperature $T_{3}$ and slip velocity $u_{s}\left(\right.$ Schaaf $\left.{ }^{(10]}\right)$ are derived as

$$
u_{s}=\frac{2-\sigma_{n}}{\sigma_{n}} \frac{2 \mu}{\rho} \sqrt{\frac{\pi}{8 R T}}\left(\frac{\partial u}{\partial n}\right)+\frac{3}{4} \frac{\mu}{\rho T}\left(\frac{\partial T}{\partial s}\right)
$$




$$
T_{s}=T_{w}+\frac{2-\sigma_{t}}{\sigma_{t}} \frac{2 \gamma}{\gamma+1} \frac{2 \mu}{\rho P_{r}} \sqrt{\frac{\pi}{8 R T}}\left(\frac{\partial T}{\partial n}\right)
$$

where $s$ is the surface tangential direction, $n$ is the inward surface normal direction, $T_{w}$ is the wall temperature. and $\sigma_{n}, \sigma_{t}$ are the surface reflection and accommodation factors, respectively. A complete accommodation, $\sigma_{n}=1$, $\sigma_{t}=1$, is assumed in this research. The slip wall conditions are implemented at each surface point implicitly. The calculated slip velocity and surface temperatures are then utilized to compute boundary fluxes. The velocity and temperature in the boundary cells are calculated as

$$
\begin{aligned}
& \vec{V}_{\text {boundary cell }}=2 \vec{V}_{\text {slip }}-\vec{V}_{\text {internal cell }} \\
& T_{\text {boundary cell }}=2 T_{s}-T_{\text {internal cell }}
\end{aligned}
$$

Since the solution methodology of BURNETT-2D is adopted from the RPLUS-2D code, detailed descriptions of the numerical algorithm can be referenced in Shuen $^{[11,12,13]}$, and Tsai and Hsieh ${ }^{[14]}$.

\section{RESULTS AND DISCUSSION}

To avoid computational complexity, the exhaust plume flow field near the afterbody of a hypersonic flight vehicle at a high altitude is computed through two steps. First, the thruster nozzle exit conditions are calculated through the Navier-Stokes solver - RPLUS2D. Secondly, the Burnett equations are solved for exhaust plume flow field near the afterbody. The computed nozzle exit conditions from the first step are averaged and used as input conditions for free stream/plume interaction. This averaging process has simplified the global computational domain, but the boundary layer effects are neglected.

Nozzle Exit Conditions Computation. The thruster nozzle has a throat diameter of 6 inches with an area ratio of 11.4 . The chamber pressure is 1600 psi and the chamber temperature is $4650 R$. An 18 step hydrogen/air chemistry model is applied to compute the nozzle internal flow field. The gas mixture is assumed to have 9 species. As altitude increases above $80 \mathrm{~km}$, the air becomes highly rarefied. Rapid expansion may occur in the base region. This highly expanded plume will interact with free stream and the flow in the forward region, which in turn, affect the nozzle exit conditions. In order to provide realistic boundary conditions at nozzle exit for the afterbody flow field calculation, flow fields in the base area and region outside the nozzle are computed simultaneously. The nozzle wall lip effects must be also taken into account in order to calculate realistic nozzle exit conditions. For preliminary study, the nozzle lip effect is neglected and the ambient free stream Mach number is set equal to 3.0 for all cases. It was assumed that no chemical reaction occurs in the base area and outside region of the nozzle, and the gas mixture contains only air. RPLLS2D code was used to compute the entire flow field. Three ambient pressures were selected for the calculation. Figures 2(a), 2(b) and 2(c) show the Mach number contours of the nozzle plume and free stream interactions for ambient pressures equal $14.7,1.0$ and $0.1 p s i$, respectively. At sea level, the typical plume expansion is shown in Figure 2(a). As altitude increases, the ambient pressure decreases. At ambient pressure equals 1.0psi, exhaust plume expands near the nozzle lip. Shock waves are formed near the edge of the plume. Further increasing altitude will generate higher expansion plume in the base area of the nozzle. Physically, the high expansion plume and the boundary layer near the nozzle lip must be calculate simultaneously during numerical iterations. However, it was neglected for simplicity. These results demonstrated that in order to obtain accurate nozzle exit conditions, base area and external free stream must be computed simultaneously with the nozzle internal flow field.

Plume Flow Field Prediction near the Aftbody Solving the Burnett Equations. A hypothetical twodimensional body is shown in Figure 3(a). A high expansion ratio thruster nozzle is placed inside the lower part of the body and used as a propulsive device. The computed nozzle exit conditions from step one are averaged and used as input conditions at the inflow boundary. Since the ambient conditions for the nozzle calculations are unknown, the results from step one can only be used as a reference. Further study is needed in order to obtain better nozzle exit conditions. Three cases were computed for exhaust plume flow field near the afterbody. The nozzle exit static pressures are specified as 5,10 and 20 times of the free stream value. Based on results from step one, it is estimated that the nozzle exit Mach number at an altitude of $80 \mathrm{~km}$ is about 3.0 for the selected nozzle configuration. Figures $3(\mathrm{a}), 3(\mathrm{~b}), 3(\mathrm{c})$ show the two-dimensional Burnett solutions of exhaust plume flow field near the afterbody of a two-dimensional flight vehicle. The free stream Mach number is 5.0 and the flight altitude is $80 \mathrm{~km}$ for all three cases. A strong oblique shock is formed near the leading edge of the flight vehicle. Exhaust plume from nozzle exit interacts with the wall and free stream to form a shockwave/viscous interaction flow pattern near the afterbody. With increasing nozzle exit pressure, the exhaust plume expands toward the upstream. It is expected that further increasing of the nozzle exit pressure will introduce a plume-induced separation near the afterbody.

\section{CONCLUSION}

A two-dimensional code to solve the Burnett equations for free stream/plume interaction near the afterbody of a hypersonic flight vehicle was developed. The computation of exhaust plume flow field near the afterbody contains two steps. The thruster nozzle exit conditions were determined a priori through a Navier-Stokes nozzle solutions which provide upstream boundary conditions for the global flow field calculation of the free stream/plume interaction. Results demonstrated that at high altitudes, the base region and outside region must be included for calculations of nozzle exit conditions si- 
multaneously. Exhaust plume flow field at an altitude of $80 \mathrm{~km}$ near the afterbody was successfully computed using the developed Burnett code. Results showed that with increasing nozzle exit pressure, the plume induced separation may occur near the afterbody which will significantly affect the aerodynamic characteristics of a hypersonic flight vehicle.

\section{REFERENCES}

[1] Fisko, K. A., and Chapman, Dean R., "Comparison of Burnett, Super-Burnett, and Monte Carlo Solutions for Hypersonic Shock Structure," In 16th International Symposium of Rarefied Gas Dynamics, 1988.

[2] Fisko, K. A., and Chapman, Dean R., "Hypersonic Shock Structure with Burnett Terms in the Viscous Stress and Heat Flux," AIAA 88-2733.

[3] Moss, J. N., Price, J. M. and Chun Ch. H., "Hypersonic rarefied Flow about a compression cornerDSMC simulation and experiment", AIAA paper 911313, 1991.

[4] Zhong, X.L., "Development and Computation of Continuum Higher Order Constitutive Relations for High-Altitude Hypersonic Flow," Ph.D. Dissertation, Stanford University, 1991.

[5] Zhong, X., MacCormack, R.W., and Chapman, Dean R., "Stabilization of the Burnett Equations and Application to High-Altitude Hypersonic Flows," AIAA Journal, Vol. 31, No. 6, June 1993.

[6] Liaw, G.S., Deng, Z.-T., Chou, L. and Mo, J.D., "The Burnett Shock Structures in Low Density Hypersonic Flows," AIAA 92-5048.
[7] Chou, L.C., Deng, Z.T. and Liaw, G.S., “ "Comparison of Shock Wave Structures by Solving Burnett and Boltzmann Equations," 6th AIA A/ASME Joint Thermophysics and Heat Transfer Conference, Colorado Springs, Colorado, June 1994. AIA A 94-2056.

[8] Deng, Zheng-Tao, Liaw, G.S. and Chou, L.C., "Numerical Investigations of Low-Density Nozzle Flow Fields by Solving Navier-Stokes and Burnett Equations," 6th AIAA/ASME Joint Thermophysics and Heat Transfer Conference, Colorado Springs, Colorado, June 1994. AIAA 94-2055.

[9] Shuen, J-S, Hsieh K-C, Tsai, Peter Y-L, and Yu, S-T, "RPLUS2D/3D USER's MANUAL," Sverdrup Technology, Inc., NASA Lewis Research Center, 1990.

[10] Schaaf, Samuel A., and Chambre, P.L., Flow of Rarefied Gases, Princeton Aeronautical Paperbacks, 8. 1961.

[11] Shuen, J-S and Yoon, S., "Numerical Study of Chemically Reacting Flows Using an LU Scheme," AIAA 88-0436.

[12] Shuen, J-S, Liou, M-S, Van Leer, B., "Inviscid Flux-Splitting Algorithms for Real Gases with NonEquilibrium Chemistry," Journal of Computational Physics, Vol. 90, pp. 371. 1990.

[13] Shuen, J-S, "Upwind Differencing and LU Factorization for Chemical Non-Equilibrium Navier-Stokes Equations," Journal of Computational Physics, Vol. 99, pp. 233-250, 1992.

[14] Tsai, Peter Y-L and Hsieh, K-C, "Comparative Study of Computational Efficiency of Two LU Schemes for Non-Equilibrium Reacting Flows." AIAA 90-0396.

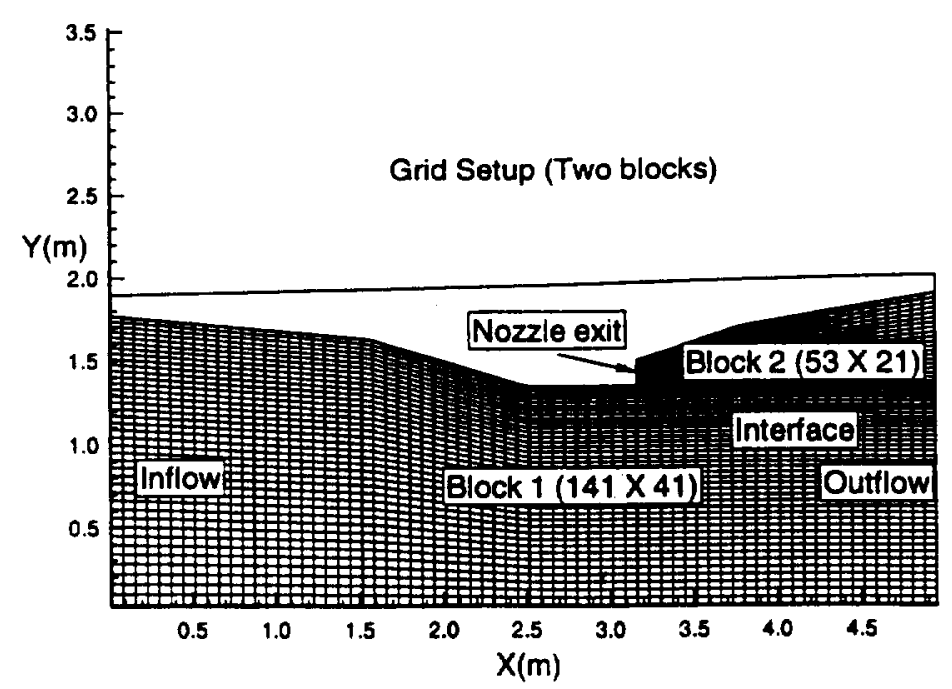

Figure 1. Schematic showing of grid setup for computation of exhaust plume flow field near the afterbody. 


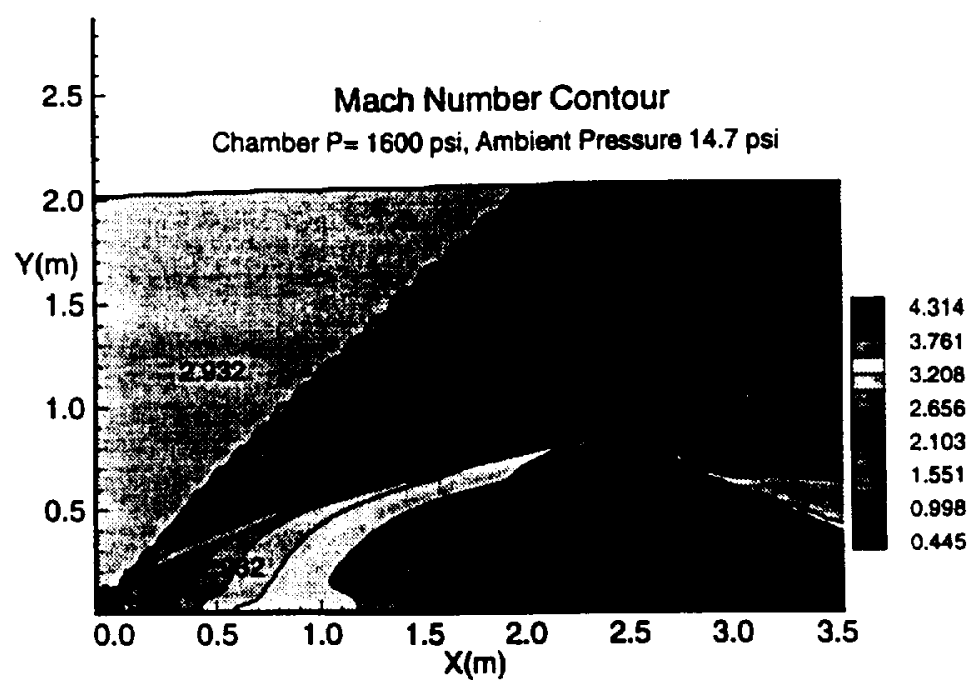

Figure 2(a). Ambient Pressure 14.7psi.

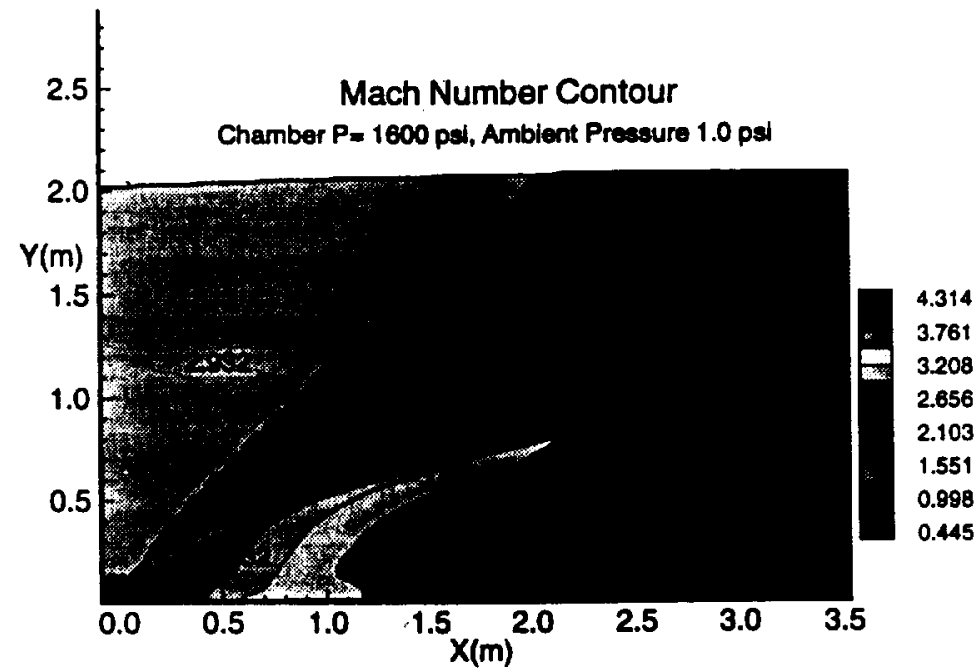

Figure 2(b). Ambient Pressure 1.0psi.

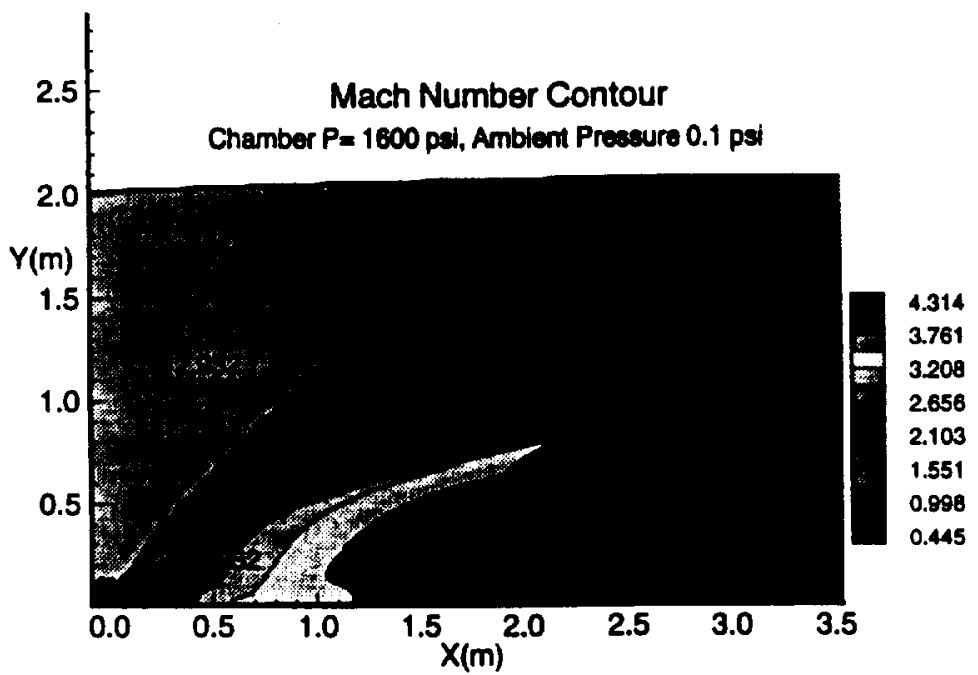

Figure 2(c). Ambient Pressure 0.1psi.

Figure 2. Mach number contours for the nozzle plume and free stream interaction. 


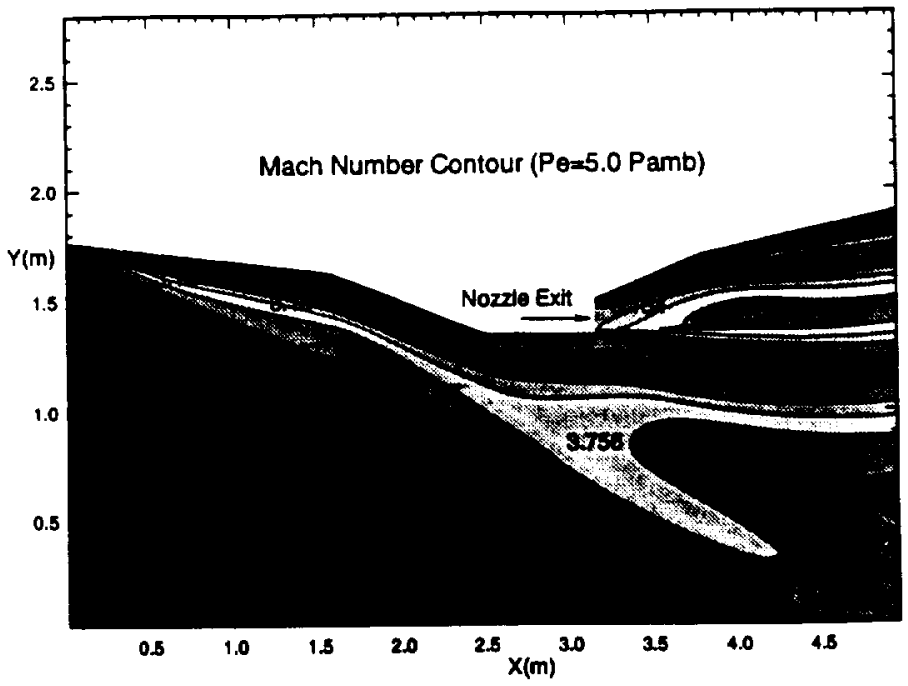

Figure 3(a). Pressure ratio $P_{e} / P_{\infty}=5$.

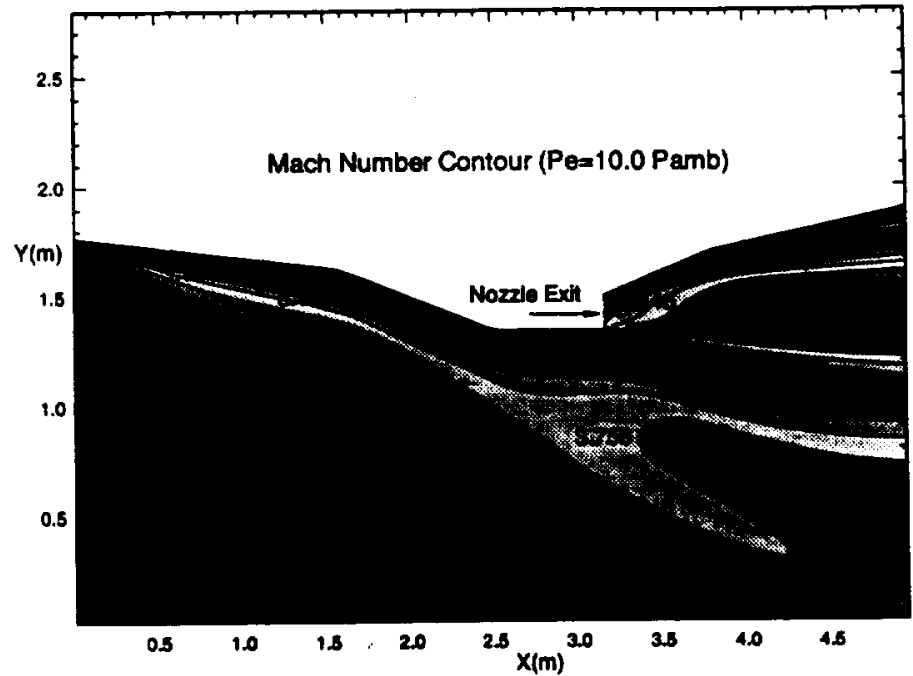

Figure 3(b). Pressure ratio $P_{e} / P_{\infty}=10$.

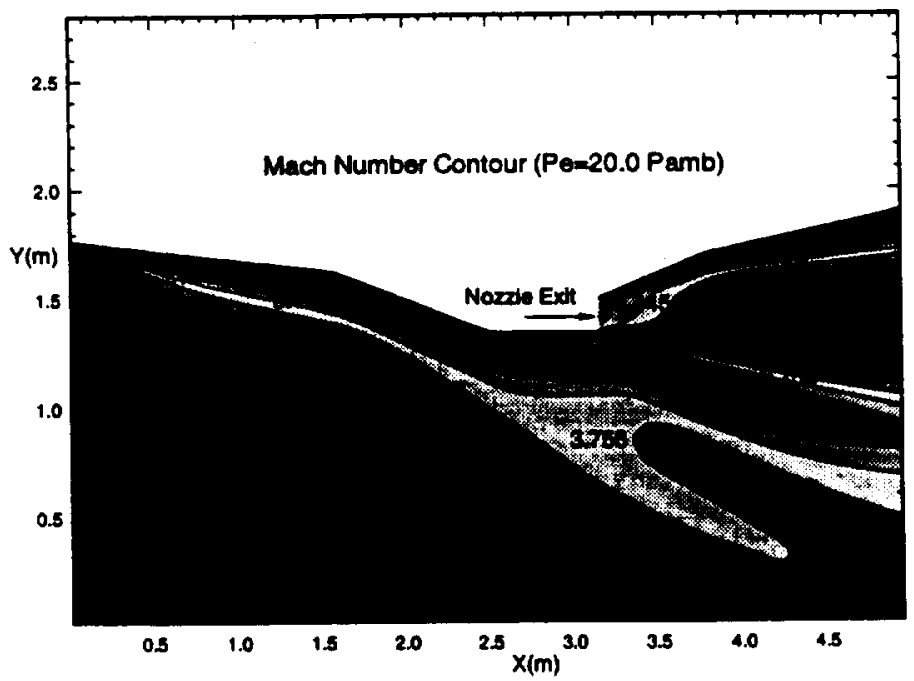

Figure 3(c). Pressure ratio $P_{e} / P_{\infty}=20$.

Figure 3. Two-dimensional Burnett solutions of exhaust plume flow field near the aftbody. 